\title{
Analysis of the effect of load application eccentricity on the stiffness of supports consisting of stack of elements
}

\author{
Krzysztof Gromysz ${ }^{1, *}$ \\ ${ }^{1}$ Silesian University of Technology, Faculty of Civil Engineering, 44-100 Gliwice, ul. Akademicka 5, Poland
}

\begin{abstract}
During the removal of building deflections, temporary supports, consisting of a stack of repeatable elements, are used. Hydraulic jacks which force the vertical displacement of the building are used, as well. Depending on the phase of deflection removal, the entire weight of the building, or part thereof, is rested on temporary supports. As a consequence of inaccuracies in the installation of a stack of elements, the building weight is transmitted onto supports in an eccentric manner. In addition, the supports are loaded with horizontal forces. The forces result from the wind load, from kinematic excitations of non-axially installed jacks, from 2nd order deformations of supports and from exceptional loads. Deformations in the vertical direction, with a positive sign, occur due to the activity of the eccentric load and horizontal forces in the supports. However, the unbounded hexagonal elements forming the stack are unable to transmit the tensile stresses. As a consequence, the stiffness of the supports depends on the load. The stiffness of temporary supports in the horizontal direction was analysed considering the existence of unintended eccentricity and the horizontal load. A support model was defined for this purpose, whose material does not transmit tensile stresses. It was shown that the scheme of the support is changing depending on the application eccentricity of the vertical load and on the horizontal load value. Eight schemes were distinguished, which may be executed by a support. It was found that if the eccentricity and the horizontal load value are increased, this will decrease the support stiffness. This stiffness varies from zero, when a rocker arm is the support scheme, to the maximum value that is corresponding to the stiffness of cantilever rod. The analysis is limited to stocky supports by assuming that the load is much smaller than critical. Moreover, the influence of the support's vertex displacement on the eccentricity was not taken into account.
\end{abstract}

\section{Introduction}

During the removal of building deflections, temporary supports, consisting of a stack of repeatable elements, are used. The elements are made of wood, steel or concrete and remain unbounded. Hydraulic jacks, which force the vertical displacement of the building, are often placed on the stacks of such elements. The use of such supports is common in the region of Silesia, where the removal of buildings deflection is carried out commonly.

A deflection is removed by the uneven raising of the structure with jacks mounted in the walls and by supporting the structure with temporary supports which form stacks of elements (Fig. 1).

When straightening the building, the supports are reinstalled many times and their length is being extended together with the uneven raising of the structure [1]. As a result of any inaccuracies in the installation of supports, an unintended eccentricity $e_{\mathrm{a}}$ of the load application occurs. At the same time, due to inaccurate installation of supports and the unavoidable rotation of the deformable subgrade, a horizontal force $Q_{x}$ occurs, which is acting on the supports. In addition, the existence of the horizontal force is caused by the activity of wind and by exceptional loads, for example due to mining tremors. The eccentric application of a vertical load and the occurrence of a horizontal load may cause deformations of the positive sign to the part of the support $(\varepsilon>0)$. Meanwhile, the supports, due to their construction, are not able to transfer normal tensile stresses. As a consequence, contact points between elements of the stack are opening along the part of the support length marked as $l_{1}$ (Fig. 2a). This length depends on the eccentricity value $e_{\mathrm{a}}$, as well as on the load $Q_{x}$. The mechanical parameters of such support are not stable, therefore.

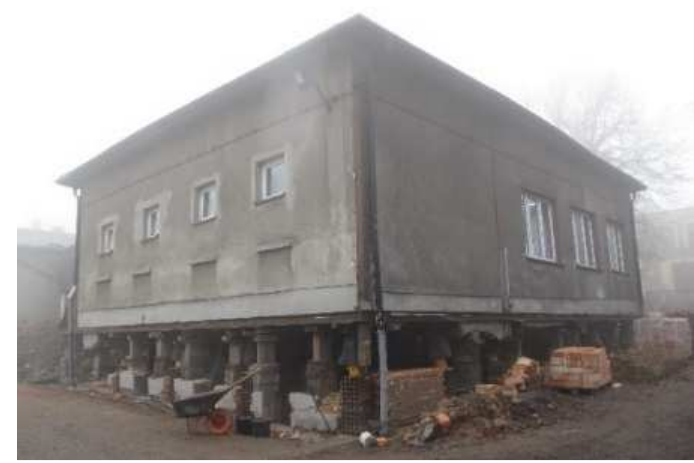

Fig. 1. Example of a building whose deflection is removed and whose weight is transmitted by supports consisting of a stack of elements.

*orresponding author: krzysztof.gromysz@polsl.pl 
The analyzes of eccentrically loaded elements have been carried out mainly in relation to reinforced concrete elements [2], steel columns [3] and masonry structures [4, 5]. Respectively, the results of the experimental research concerns mainly GFRP Reinforced Geopolymer Concrete [6], steel [3] and masonry structures [7, 8]. However, the possibility of transferring eccentrically applied forces by temporary supports consisting of a stack of elements has not been studied. Meanwhile, the possibility of transferring an eccentrically applied load by these supports determines the safety of the building deflection removal process. The mechanical parameters of supports need to be known when designing the straightening of a building.

Support stiffness in the horizontal direction is analysed below with an analytical model. The stiffness is defined as:

$$
k_{x, l}=\frac{\Delta Q_{x}}{\Delta u_{x, l}}
$$

where $\Delta Q_{x}$ is an increment of the horizontal force applied to the support at the height $l$, and $\Delta u_{x, l}$ is a value of support displacement at the same height in the $\Delta Q_{x}$ direction. This article is limited to analysing the effect of 1 st order deformation on support stiffness (Fig. 2b). It was therefore assumed that the force value $Q_{z}$ is much smaller than a critical load and the influence of the support vertex $(z=l)$ displacement on the increase of deformation was omitted. a)

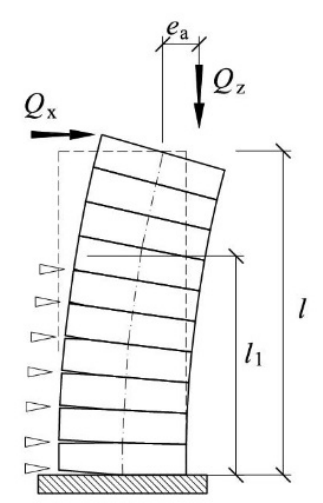

b)

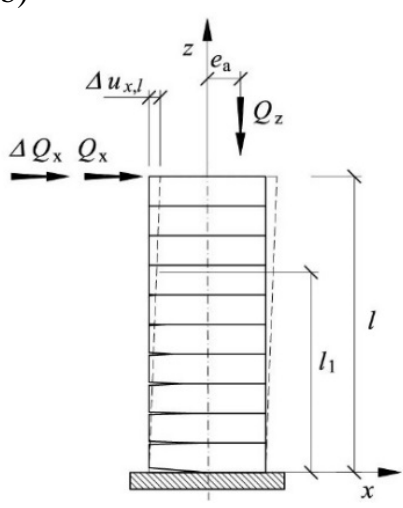

Fig. 2. Temporary support consisting of a stack of elements: a) scheme of deformations caused by the unintended eccentricity $e_{\mathrm{a}}$ and by the horizontal load $\left.Q_{x}, \mathrm{~b}\right)$ scheme for determination of the stiffness $k_{x, l}$; a place is marked with arrows where contact points between elements are opened

In practical solutions, a cross-section of temporary supports is usually rectangular (Fig. 3 a,b), although supports with a circular section are also used (Fig. 3 c,d). Repeatable elements of supports are made of wood (Fig. 3 a,d), steel (Fig. 3 b) or of composite steel and concrete elements (Fig. 3 c). It is therefore necessary to determine general dependencies for the stiffness of such supports depending on the load application eccentricity and the value of horizontal force. The deliberations in the article are limited to a support with rectangular section with the width $b$ and height $h$.

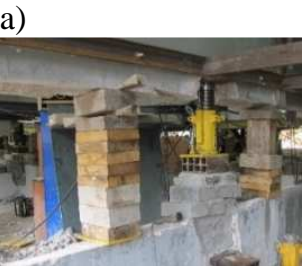

c)

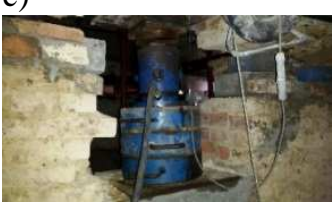

b)

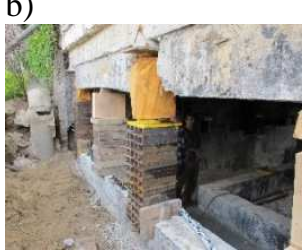

d)

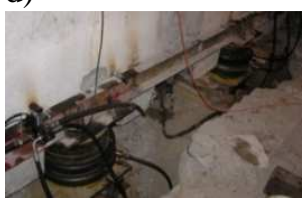

Fig. 3. Examples of temporary supports: a) support consisting of wooden hexagonal elements, b) support placed on steel hexagonal elements, c), d) supports with circular section

\section{Support model}

A model of the support consists of a rod with the length $l$, having the cross section with the height $h$ and width $b$. The rod axis is marked with $z$, whereas the direction perpendicular to the road axis is marked with $x$. The beginning of the coordinate system $(x, z)$ is situated in the place where the rod is supported. The vertex of the rod is free and may move towards the direction $x$. The boundary conditions of the supported end of the rod prevent its displacement. The possible rotation of the part of or of the entire rod section in the coordinate $z=0$ depends on the distribution of stresses in the base. The vertex of the rod $(z=l)$ is loaded with the vertical force $Q_{z}$ applied in the distance $e_{\mathrm{a}}$ to the road axis (unintended eccentricity) and with the horizontal force $Q_{x}$. The unintended eccentricity $e_{\mathrm{a}}$ assumes a negative value if the force $Q_{z}$ is applied on the left side of the axis $z$, or a positive value, in the opposite case. The force $Q_{x}$, directed to the right side, is considered in this paper, hence $Q_{x}$ assumes positive values.

\subsection{Assumptions of the model}

A load applied to the model causes vertical stresses in the material of supports, marked as $\sigma_{\mathrm{z}}$. (Fig. 4). In practice, the value of such stresses is smaller than the strength of the material of repeatable elements of the stack; it was therefore assumed in the model that deformations of such elements are elastic.

The model of the material is assumed which does not transmit stresses, with positive values towards the axis $z$. Moreover, a linear dependence is assumed between the stress $\sigma_{\mathrm{z}}$ and the longitudinal deformation $\varepsilon$, with a negative mark, and that the modulus of elasticity of support's material equals $E$. For this reason, the value of stresses $\sigma_{z}$ is:

$$
\begin{array}{cc}
\sigma_{z}=0 & \varepsilon \geq 0 \\
\sigma_{z}<0 & \varepsilon<0
\end{array}
$$

As mentioned above, the analysis is limited to first order deformations. Hence, the rod stiffness in the horizontal direction, defined with the formula (1), can be calculated from the dependency: 


$$
k_{x, l}=\frac{1}{\delta}
$$

where $\delta$ is the displacement caused by the unit force applied to the rod vertex and acting in the horizontal direction.

Shear and normal stresses $\sigma_{z}$ are caused in the base of the support by external loads. The location of the resultant of normal stresses on the lower base of the supports is determined from the dependency:

$$
e_{\mathrm{d}}=\eta \cdot l+e_{\mathrm{a}}
$$

where $\eta$ is the quotient of value of the force $Q_{x}$ by $Q_{z}$. This quotient has always a non-negative value, because the analysed force $Q_{x}$ has always a positive sense:

$$
\eta=\frac{Q_{x}}{Q_{z}} \geq 0
$$

It was assumed that there is no mutual slide between the elements making the stack, because friction stresses are caused on the surface of the elements, which are balancing the effect of the horizontal force. This is equivalent to the assumption that the value $\eta$ is smaller than the friction coefficient of the elements of supports.

The eccentricity value meets the condition:

$$
-\frac{h}{2}<e_{\mathrm{a}}<\frac{h}{2}
$$

The height of the cross-section zone, where the deformations $\varepsilon<0$ exist, is marked with $h_{1}$, and is called the height of the compressed zone or the mating height. If the whole cross-section of the rod is being compressed, then $h_{1}=h$. In case where positive deformations exist in the cross-section of the rod, $h_{1}<h$. This means that the height of the compressed zone meets the condition:

$$
0<h_{1} \leq h
$$

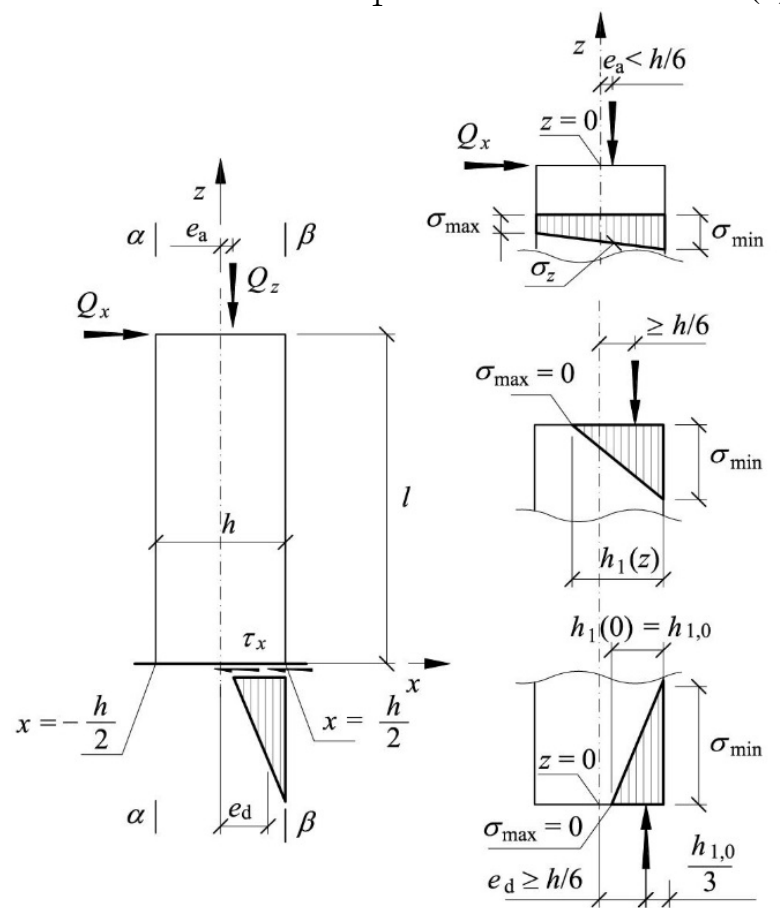

Fig. 4. Scheme of the support and examples of possible distribution of normal stresses $\sigma_{z}$

The limit value of the horizontal load $Q_{x}$, with the given eccentricity $e_{\mathrm{a}}$, is determined from the condition $h_{1} \rightarrow 0$ in the cross-section at the height $z=0$. This means that the condition is met:

$$
\eta=\frac{\frac{h}{2}-e_{\mathrm{a}}}{l}
$$

The distribution of normal stresses $\sigma_{\mathrm{z}}$ in the crosssection of the rod, by assuming the constant modulus of elasticity $E$, is trapezoid-shaped if the compression zone $h_{1}=h$, or triangle-shaped when $h_{1}<h$ (Fig. 4).

A location of resultant stresses $\sigma_{\mathrm{z}}$ in relation to the vertical axis, at the height $z=0$, is marked as $e_{\mathrm{d}}$ (Fig. 4). The height of the compressed cross-section zone, in the place where the rod is supported $h_{1}(z=0)=h_{1,0}$, depends on the value of this eccentricity and is:

$$
h_{1,0}=\left\{\begin{array}{ccc}
3\left(\frac{h}{2}-\left|e_{\mathrm{d}}\right|\right) & \text { for } & \left|e_{\mathrm{d}}\right|<\frac{h}{6} \\
h & \text { for } & \left|e_{\mathrm{d}}\right| \geq \frac{h}{6}
\end{array}\right.
$$

\subsection{Distributions of normal stresses}

The value of the unintended eccentricity $e_{\mathrm{a}}$, and the distribution of normal stresses on the lower surface of the support model, have all influence on the distribution of stresses $\sigma_{z}$ at the height $z$. Eight such distributions are possible for the assumptions made. Such distributions are defining eight possible schemes of the support, which are marked as $A, B, C, D 1, D 2, E 1, E 2, F$ (Fig. 5). Parts of the lengths of the supports, where $h_{1} \neq h$, are marked as $l_{1, \mathrm{~g}}$, if positive deformations exist in the upper part of the support model, and as $l_{1, \mathrm{~d}}$, if positive deformations exist in the lower part of the model. Along such lengths, the mating heights of the cross-section $h_{1, \mathrm{~g}}(z)$ and $h_{1, \mathrm{~d}}(z)$ are the linear functions of the coordinate $z$. For this reason, also the moments of inertia $I_{1, \mathrm{~g}}(z)$ and $I_{1, \mathrm{~d}}(z)$ of the mating crosssection along such sections, represent the linear function $z$. Hence, the displacement $\delta$, in the direction $x$, caused by the unit force, can be calculated from the following general dependency:

$$
\begin{gathered}
\delta=\frac{1}{E} \int_{0}^{l_{1, \mathrm{~d}}} \frac{(l-z)^{2}}{I_{1, \mathrm{~d}}(z)} \mathrm{d} z+\frac{1}{E} \int_{l_{\mathrm{d}}}^{l-l_{1, \mathrm{~g}}} \frac{(l-z)^{2}}{I_{1, \mathrm{~d}}(z)} \mathrm{d} z \\
+\frac{1}{E} \int_{l-l_{1, \mathrm{~g}}}^{l} \frac{(l-z)^{2}}{I_{1, \mathrm{~d}}(z)} \mathrm{d} z
\end{gathered}
$$

If $l_{1, \mathrm{~g}}=l_{1, \mathrm{~d}}=0$, then $\delta=l^{3} / 3 E I$, and the stiffness (3) of the support is $k_{x, l}=3 E I / l^{3}$, and corresponds to the stiffness of the cantilever rod.

Two edges are distinguished in the models: $\alpha$ corresponding to the coordinate $x=-h / 2$ and $\beta$ corresponding to the coordinate $x=h / 2$ (Fig. 4).

The condition of zeroing of stresses $\sigma_{z}$ on the edge $\alpha$ on the height $z$ is:

$$
\frac{Q_{x}(l-z)}{W}+\frac{Q_{z} e_{\mathrm{a}}}{W}-\frac{Q_{z}}{F}=0
$$

where $W$ and $F$ are, respectively, the section modulus and the field of the compressed cross-section of the support model on the height $z$. If $h_{1}=h$, then we have:

$$
W=\frac{b \cdot h}{6} ; F=b \cdot h
$$


On the other hand, the condition of zeroing of stresses $\beta$ is:

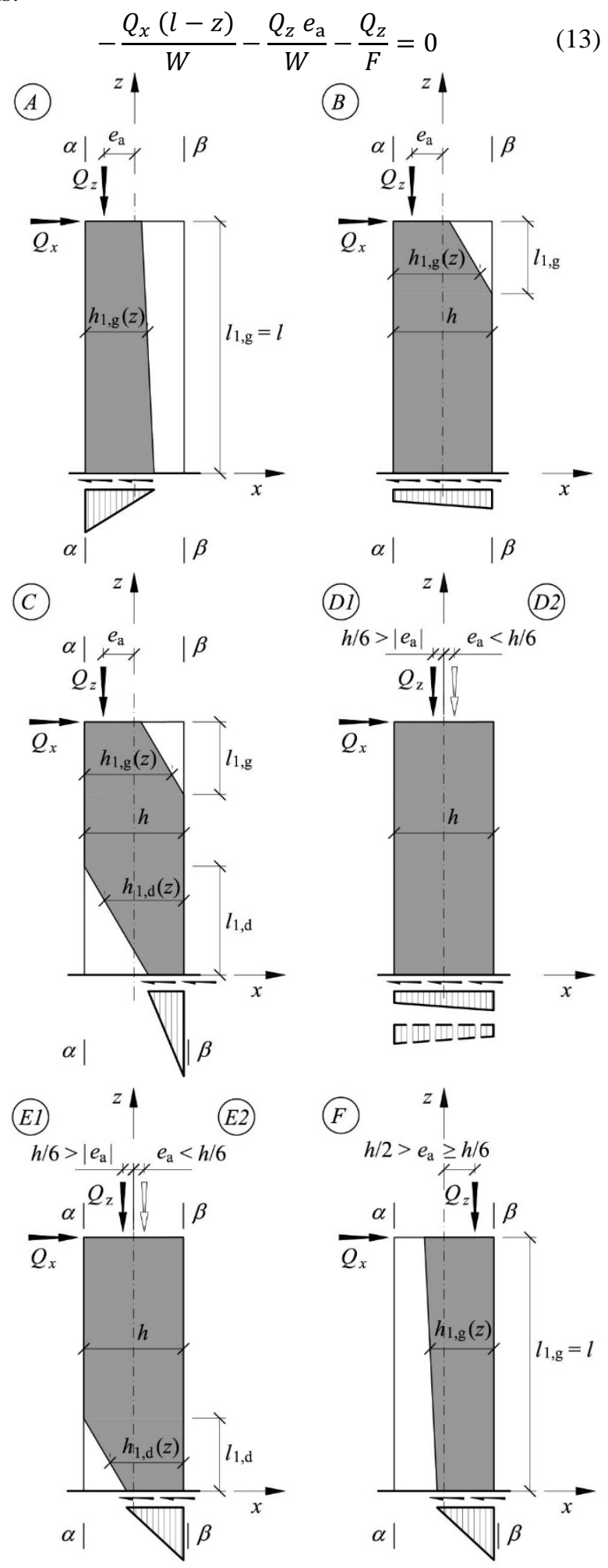

Fig. 5. Schemes of supports $(A, B, C, D 1, D 2, E 1, E 2, F)$ depending on the value of the unintended eccentricity $e_{\mathrm{a}}$ and the horizontal load $Q_{x}$.

\subsubsection{Scheme A}

A condition for executing the scheme $A$ (Fig. 5) is to apply the force $Q_{z}$ outside the core of the cross-section, i.e.:

$$
-\frac{h}{2}<e_{\mathrm{a}} \leq-\frac{h}{6}
$$

and also the existence, along the whole length $l$ of the edge $\beta$, of the positive deformations $\varepsilon$. A limit situation in the analysed scheme $A$ occurs when the distribution of stresses at the height $z=0$ is triangular and covers its entire height, this means $h_{1,0}=h$. On the basis of (13) and (12) and (5), an allowable range of changes in the value $\eta$ in the scheme $A$ was determined:

$$
0<\eta \leq \frac{\left|e_{\mathrm{a}}\right|-\frac{h}{6}}{l}
$$

The mating height of the cross-section $h_{1,0}$, based on (4) and (9), is:

$$
h_{1,0}=3\left(\frac{h}{2}+\eta \cdot l-\left|e_{\mathrm{a}}\right|\right)
$$

The mating height in the region of the support vertex, by analogy to (9), was assumed as equivalent to:

$$
h_{1, l}=3\left(\frac{h}{2}-\left|e_{\mathrm{a}}\right|\right)
$$

The dependency on the mating height was established based on (16) and (17):

$$
h_{1, \mathrm{~g}}(\mathrm{z})=3 h\left(\frac{1}{2}-\frac{\left|e_{\mathrm{a}}\right|}{h}+\eta \frac{l-z}{h}\right)
$$

\subsubsection{Scheme $B$}

A condition for the scheme $B$ to occur is to apply the load $Q_{z}$ outside the core of the cross-section (condition (14)), and the existence of the trapezoid distribution of stresses $\sigma_{z}$ for $z=0$. A limit situation occurs when the stress value $\sigma_{z}$ on the edge $\beta$ in $z=0$ is zero. This condition was recorded by substituting in (13) $z=0$, and then the maximum value $\eta$ was determined from it. In view of the above, the condition for executing the scheme $B$ is:

$$
0 \leq \eta \leq \frac{\left|e_{\mathrm{a}}\right|+\frac{h}{6}}{l}
$$

The length $l_{1, \mathrm{~g}}$ of the support, where the height of the cross-section $h_{1}<h$ exists, is determined from the condition of zeroing of stresses on the edge $\beta$. After substituting in (13) $z=l_{1, \mathrm{~g}}$ and after considering (12), the following is obtained:

$$
l_{1, \mathrm{~g}}=l_{1, \mathrm{~g}}=\frac{1}{\eta}\left(\left|e_{\mathrm{a}}\right|-\frac{h}{6}\right)
$$

Considering the expressions (17) for $h_{1, l}$ and (20) for $l_{1, \mathrm{~g}}$, the cross-section height $h_{1, \mathrm{~g}}$ was determined along the length $l_{1, \mathrm{~g}}$ :

$$
h_{1, \mathrm{~g}}(\mathrm{z})=3 h\left(\frac{1}{2}-\frac{\left|e_{\mathrm{a}}\right|}{h}+\eta \frac{l-z}{h}\right)
$$

\subsubsection{Scheme C}

The scheme $C$ of the support is executed if the eccentricity $e_{\mathrm{a}}$ has a negative value and its absolute value is higher than $h / 6$, and the distribution of stresses $\sigma_{z}$ in the base of the model $(z=0)$ is triangular, whereas the extreme value of the stresses, $\sigma_{z}$, exists on the edge $\beta$. The consequence of such application of the load and of the distribution of stresses in $z=0$ is the existence of positive 
deformations on the edge $\alpha$ at the length $l_{1, \mathrm{~d}}$ and on the edge $\beta$ at the length $l_{1, \mathrm{~g}}$. The minimum value of the quotient $\eta$ was determined from the zeroing condition of the stresses $\sigma_{z}$ on the edge $\alpha$ in the base of the support. The following is obtained by substituting $z=0$ in the equation (11):

$$
\eta_{\min }=\frac{\left|e_{\mathrm{a}}\right|+\frac{h}{6}}{l}
$$

The maximum value of the quotient $\eta$, hence the maximum value of the force $Q_{x}$ was determined from the condition $h_{1,0} \rightarrow 0$, which is equivalent to $e_{\mathrm{d}} \rightarrow h / 2$. The following was obtained from the condition of balance of bending moments of the rod:

$$
\eta_{\text {max }}=\frac{\left|e_{\mathrm{a}}\right|+\frac{h}{2}}{l}
$$

The length $l_{1, \mathrm{~d}}$ was determined from the zeroing condition of stresses on the edge $\alpha$. The following was obtained by substituting in (11) $z=l_{1, \mathrm{~d}}$ :

$$
l_{1, \mathrm{~d}}=l-\frac{1}{\eta}\left(\left|e_{\mathrm{a}}\right|+\frac{h}{6}\right)
$$

The maximum length $l_{1, \mathrm{~d}}\left(l_{1, \mathrm{~d}, \max }\right)$ was determined by substituting in (24) - instead of $\eta$ - the value $\eta_{\max }$, described with the formula (23). Hence:

$$
l_{1, \mathrm{~d}, \max }=\frac{2}{3} l\left(\frac{h}{2\left|e_{\mathrm{a}}\right|+h}\right)
$$

By substituting, in the equation (9), the dependency (4), in the case $\left|e_{\mathrm{d}}\right|<h / 6$, the height $h_{1,0}$ was determined as equal to:

$$
h_{1,0}=3\left(\frac{h}{2}+\left|e_{\mathrm{a}}\right|-\eta \cdot l\right)
$$

The following was determined using the dependencies (26) and (24):

$$
h_{1}(\mathrm{z})=3 h\left(\frac{1}{2}+\frac{\left|e_{\mathrm{a}}\right|}{h}-\frac{l-z}{h}\right)
$$

The length $l_{1, \mathrm{~g}}$ and height $h_{1, \mathrm{~g}}$ of the cross-section is determined the same as in the scheme $B$. For this reason, in the scheme $C$, the dependencies (20) and (21) for $l_{1, \mathrm{~g}}$ and $h_{1, \mathrm{~g}}$ are applicable.

\subsubsection{Schemes D1 and D2}

The schemes $D 1$ and $D 2$ are executed in the situation where negative deformations in the direction $z$ exist in the whole support. A prerequisite for such a situation is that the force $Q_{z}$ is applied in the core of the cross-section. The limit values $\eta$ are determined from the zeroing condition of the stresses $\sigma_{z}$ in $z=0$ on the edge $\alpha$.

A pre-condition for the existence of the scheme $D 1$ is the occurrence of eccentricity $e_{\mathrm{a}}$, with a negative value:

$$
-\frac{h}{6}<e_{\mathrm{a}} \leq 0
$$

and that the following condition is met by the quotient $\eta$ :

$$
0 \leq \eta \leq \frac{\frac{h}{6}+\left|e_{\mathrm{a}}\right|}{l}
$$

The scheme $D 2$ is executed if the eccentricity $e_{\mathrm{a}}$ assumes a negative value:

$$
0 \leq e_{\mathrm{a}}<\frac{h}{6}
$$

and the quotient $\eta$ meets the condition:

$$
0 \leq \eta<\frac{\frac{h}{6}-\left|e_{\mathrm{a}}\right|}{l}
$$

\subsubsection{Schemes E1 and E2}

The schemes $E 1$ and $E 2$ occur if the eccentricity $e_{\mathrm{a}}$ meets, respectively, the conditions (28) and (30) and, at the same time, the conditions (29) and (31) are not met. In such situation, the distribution of stresses $\sigma_{z}$ in $z=0$ assumes the triangular distribution. The dependencies for the maximum values $\eta$ are obtained from the zeroing condition of the stresses $\sigma_{z}$ on the edge $\alpha$ in $z=0$. The minimum values $\eta$ in the schemes $E 1$ and $E 2$ are equivalent to maximum values from the schemes $D l$ and $D 2$. In connection with the above, the condition for the value $\eta$ in the scheme $E 1$, is:

$$
\frac{\left|e_{\mathrm{a}}\right|+\frac{h}{6}}{l} \leq \eta \leq \frac{\left|e_{\mathrm{a}}\right|+\frac{h}{2}}{l}
$$

An expression for the length $l_{1, \mathrm{~d}}$ was obtained from the zeroing condition of the stresses $\sigma_{z}$ on the edge $\alpha$ in $z=l$ $-l_{1, \mathrm{~d}}$ :

$$
l_{1, \mathrm{~d}}=l-\frac{1}{\eta}\left(\frac{h}{6}+\left|e_{\mathrm{a}}\right|\right)
$$

The height $h_{1,0}$ of the cross-section transmitting the loads in $z=0$ is, based on the conditions (9) and (4):

$$
h_{1,0}=3\left(\frac{h}{2}-\eta l+\left|e_{\mathrm{a}}\right|\right)
$$

and the value $h_{1, \mathrm{~d}}$, for $z$ changing from zero to $l_{1, \mathrm{~d}}$, equals:

$$
h_{1, \mathrm{~d}}=3 h\left(\frac{1}{2}+\frac{\left|e_{\mathrm{a}}\right|}{h}-\eta \frac{l-z}{h}\right)
$$

An analogous condition for execution of the scheme $E 2$ is:

$$
\frac{\frac{h}{6}-e_{\mathrm{a}}}{l} \leq \eta \leq \frac{\frac{h}{2}-e_{\mathrm{a}}}{l}
$$

and the length $l_{1, \mathrm{~d}}$ is:

$$
l_{1, \mathrm{~d}}=l-\frac{1}{\eta}\left(\frac{h}{6}-\left|e_{\mathrm{a}}\right|\right)
$$

The mating height $h_{1,0}$, based on the conditions (9) and (4), is:

$$
h_{1,0}=3\left(\frac{h}{2}-\eta l-\left|e_{\mathrm{a}}\right|\right)
$$

and the value $h_{1, \mathrm{~d}}$, for $z$ changing from zero to $l_{1, \mathrm{~d}}$, is described with the dependency:

$$
h_{1, \mathrm{~d}}(z)=3 h\left(\frac{1}{2}-\frac{e_{\mathrm{a}}}{h}-\eta \frac{l-z}{h}\right)
$$

\subsubsection{Scheme $F$}

The last possible scheme, which can execute the support model with the positive value of the force $Q_{x}$, is the scheme $F$ (Fig. 5). It is characterised by the fact that deformations with positive values occur along the whole length of the edge $\alpha$. The above results from the condition:

$$
\frac{h}{6} \leq e_{\mathrm{a}}<\frac{h}{2}
$$


and from the triangular distribution of stresses $\sigma_{z}$ in $z=0$. A minimum value of the parameter $\eta$ is zero, and a maximum value results from the fact that $e_{\mathrm{d}}$ has reached the value $h / 2$. In connection with the above:

$$
0 \leq \eta \leq \frac{\frac{h}{2}-e_{\mathrm{a}}}{l}
$$

The height $h_{1,0}$ of the mating cross-section in $z=0$ is, based on the conditions (9) and (4), computed as:

$$
h_{1,0}=3\left(\frac{h}{2}-\eta l-\left|e_{\mathrm{a}}\right|\right)
$$

and the value $h_{1, \mathrm{~d}}$, for $z$ changing from zero to $l_{1, \mathrm{~d}}$, is described with the dependency:

$$
h_{1, \mathrm{~d}}(z)=3 h\left(\frac{1}{2}-\frac{e_{\mathrm{a}}}{h}-\eta \frac{l-z}{h}\right)
$$

The conditions for the value of the eccentricity $e_{\mathrm{a}}$ and of the quotient $\eta$ in case of particular schemes, is summarised in table 1.

On the other hand, the dependencies introduced above, on the length $l_{1, \mathrm{~g}}$ and $l_{1, \mathrm{~d}}$ and the height $h_{1, \mathrm{~g}}$ and $h_{1, \mathrm{~d}}$, are summarised for particular schemes in table 2 .

Table 1. Conditions for $e_{\mathrm{a}}$ and $\eta$ in schemes

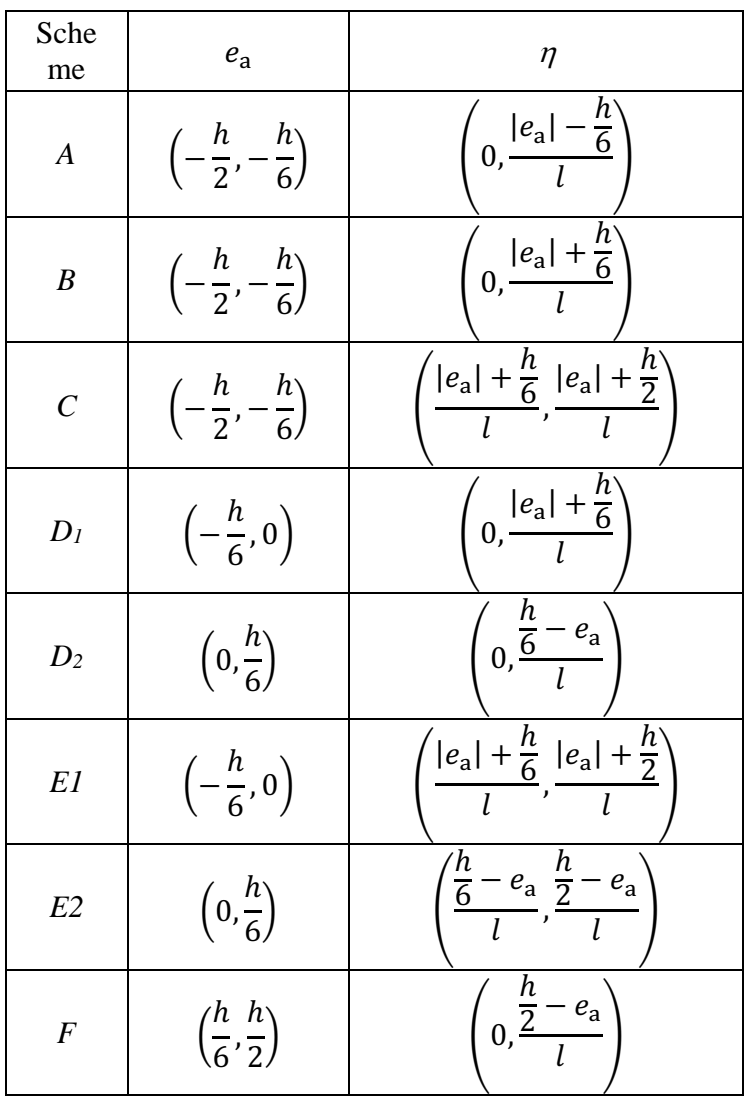

Table 2. Dependences on $l_{1}$ and $h_{1}$ for schemas

\begin{tabular}{|c|c|c|}
\hline Sch. & $l_{1}$ & $h_{1}(z)$ \\
\hline$A$ & $l_{1}=l$ & $3 h\left(\frac{1}{2}-\frac{\left|e_{\mathrm{a}}\right|}{h}+\eta \frac{l-z}{h}\right)$ \\
\hline$B$ & $\begin{array}{l}l_{1, \mathrm{~g}} \\
=\frac{1}{\eta}\left(e_{\mathrm{a}}-\frac{h}{6}\right)\end{array}$ & $3 h\left(\frac{1}{2}-\frac{\left|e_{\mathrm{a}}\right|}{h}+\eta \frac{l-z}{h}\right)$ \\
\hline
\end{tabular}

\begin{tabular}{|c|l|c|}
\hline \multirow{2}{*}{$C$} & $\begin{array}{l}l_{1, \mathrm{~g}} \\
=\frac{1}{\eta}\left(e_{\mathrm{a}}-\frac{h}{6}\right)\end{array}$ & $\begin{array}{l}h_{1, \mathrm{~g}} \\
=3 h\left(\frac{1}{2}-\frac{\left|e_{\mathrm{a}}\right|}{h}+\eta \frac{l-z}{h}\right)\end{array}$ \\
\cline { 2 - 3 } & $\begin{array}{l}l_{1, \mathrm{~d}} \\
=l \\
-\frac{1}{\eta}\left(e_{\mathrm{a}}+\frac{h}{6}\right)\end{array}$ & $\begin{array}{c}h_{1, \mathrm{~d}} \\
=3 h\left(\frac{1}{2}+\frac{\left|e_{\mathrm{a}}\right|}{h}-\eta \frac{l-z}{h}\right)\end{array}$ \\
\hline$D 1$ & 0 & $h$ \\
\hline$D 2$ & 0 & $3 h\left(\frac{1}{2}+\frac{\left|e_{\mathrm{a}}\right|}{h}-\eta \frac{l-z}{h}\right)$ \\
\hline$E 1$ & $\begin{array}{l}l_{1, \mathrm{~d}} \\
-\frac{1}{\eta}\left(\frac{h}{6}+e_{\mathrm{a}}\right)\end{array}$ & $3 h\left(\frac{1}{2}-\frac{e_{\mathrm{a}}}{h}-\eta \frac{l-z}{h}\right)$ \\
\hline$E 2$ & $\begin{array}{l}l_{1, \mathrm{~d}} \\
=l\end{array}$ & $3 h\left(\frac{1}{2}-\frac{e_{\mathrm{a}}}{h}-\eta \frac{l-z}{h}\right)$ \\
\hline$F$ & $\left.-\frac{h}{\eta}-e_{\mathrm{a}}\right)$ & $l_{1}=l$
\end{tabular}

\section{Examples of stiffness calculation}

The dependencies for $h_{1, \mathrm{~g}}$ and $h_{1, \mathrm{~d}}$ listed in table 2 allow to determine the moments of inertia of the mating crosssections $I_{1, \mathrm{~g}}(z)$ and $I_{1, \mathrm{~d}}(z)$ at the lengths $l_{1, \mathrm{~g}}$ and $l_{1, \mathrm{~d}}$. When knowing such dependencies, one can use, in the adopted model, the formulas (10) and (3) for the value $\delta$ and stiffness $k_{x, l}$.

When applying below the model defined, the following was analysed: the effect of unintended eccentricity $e_{\mathrm{a}}$ of the application of the vertical force $Q_{z}$ and of the occurrence of the horizontal load $Q_{x}$ on the stiffness $k_{x, l}$.

\subsection{The effect of the change of eccentricity $e_{a}$ on support stiffness}

The subject of the analysis is an analytical model of a support having the length $l=1.0 \mathrm{~m}$ and the rectangular cross-section with the width $b$ and height $h$. The stiffness $k_{x, l}$ was determined for this model depending on the eccentricity $e_{\mathrm{a}}$, changing in the range of:

$$
-\frac{h}{2}<e_{\mathrm{a}}<\frac{h}{2}
$$

Three permanent values $\eta$ of $0,0.1$ and 0.2 were adopted for the calculations. The results obtained were presented as charts provided in figure 6 . On such charts, the quotient $e_{\mathrm{a}} / h$ was provided on the horizontal axis, and the stiffness $k_{x, l}$ on the vertical axis, divided by $E I$, where $E I$ is the flexural rigidity of rectangular section $b / h$. It is worth-noting that the stiffness of the support whose all cross-sections are compressed is $3 E I / l^{3}$, which, with the assumed $l=1$, equals $3 E I$. It is also the highest value of the stiffness $k_{x, l}$.

With the lack of a horizontal load $(\eta=0)$ and eccentricity $e_{\mathrm{a}}$, varying in the range of $-h / 6$ to $h / 6$, the stiffness $k_{x, l}$ of the support is constant and corresponds to a cantiliver rod with the constant stiffness of the crosssection to bending of EI. This range is outside the 
horizontal line for figure $6 \mathrm{a}$ and covers two schemes of the support: $D 1$, when $-h / 6<e_{\mathrm{a}}<0$, and $D 2$ when $0<e_{\mathrm{a}}$ $<h / 6$. The growth of the absolute value $e_{\mathrm{a}}$ causes the stiffness $k_{x, l}$ to fall to the zero value, when $e_{\mathrm{a}} \rightarrow h / 2$. When $-h / 2<e_{\mathrm{a}}<-h / 6$, the support is executing the scheme $A$, and when $-h / 6<e_{\mathrm{a}}<-h / 2$, the support is executing the scheme $F$.

a)

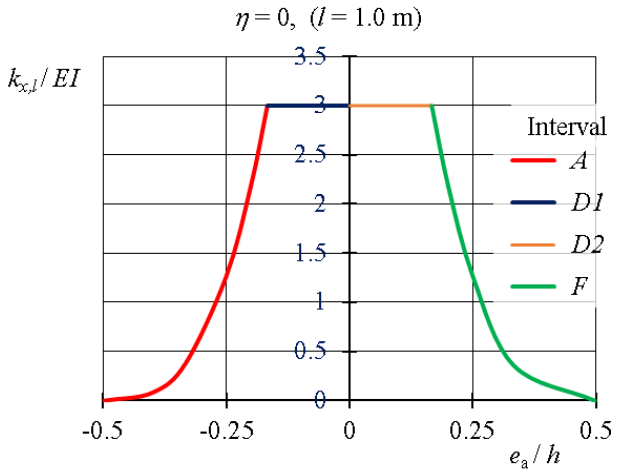

b)

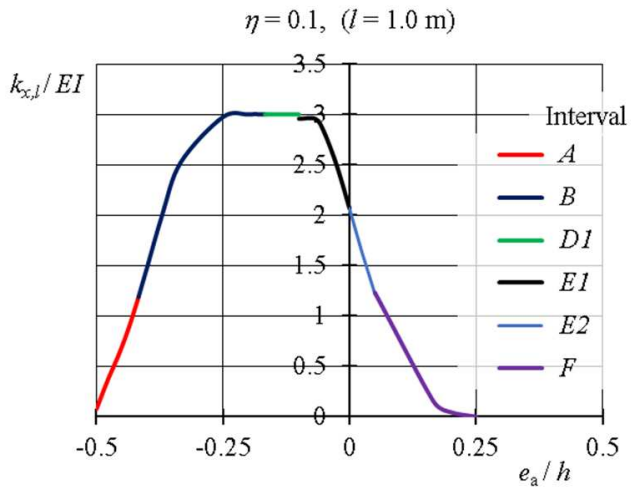

c)

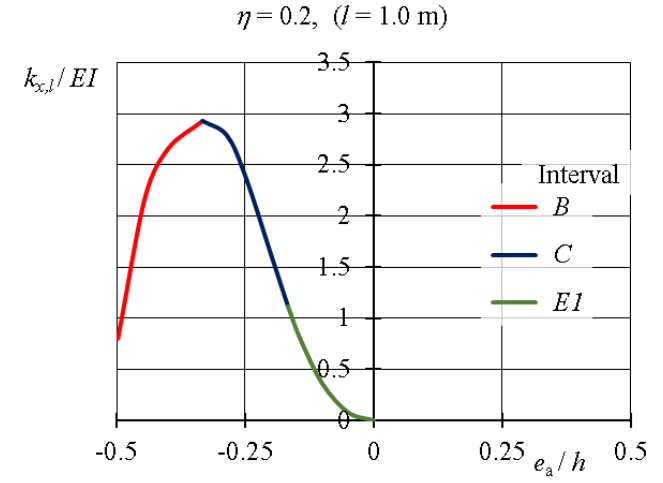

Fig. 6. Support stiffness $k_{x, l}$, as a value dependent on eccentricity $e_{\mathrm{a}}$, calculated for: a) $\eta=0$, b) $\eta=0.1$, c) $\eta=0.2$.

Figure $6 \mathrm{~b}$ shows the results of a calculation analysis of changes in the stiffness $k_{x, l}$ of the support for $\eta=0.1$ and eccentricity of $e_{\mathrm{a}}$ changing between $-h / 2$ to $h / 2$. It was found that - along with the change of the eccentricity - the support is executing schemes: $A, B, D 1, E 1, E 2$ and $F$. The support model is characterised by maximum stiffness, corresponding to the cantilever rod, only in the range $e_{\mathrm{a}}$ changing between $-h / 6$ and $-h / 10$. The support reaches the zero stiffness with the eccentricity of $e_{\mathrm{a}}=h / 4$.

Figure $6 \mathrm{c}$ shows the results of a calculation analysis of changes in the stiffness $k_{x, l}$ of the support for $\eta=0.2$ and eccentricity of $e_{\mathrm{a}}$ changing between $-h / 2$ to $h / 2$. It was found that - along with the change of the eccentricity - the support is executing three schemes: $B, C$ and $E 1$. For $e_{\mathrm{a}}=$
- $h / 2$, the support stiffness is different than zero. On the other hand, $k_{x, l}$ reaches the value of zero already for $e_{\mathrm{a}}=$ 0 . For $\eta=0.2$, the support - for neither of the eccentricity values - does not reach the stiffness corresponding to the cantilever rod with constant stiffness of the cross-section of $E I$.

\subsection{Effect of the horizontal load value on support stiffness}

A calculation analysis of the effect of horizontal load on support stiffness was carried out. The analysis' results are shown in the figures (Fig. 7 and Fig. 8a,b), where, for the constant value of the quotient $e_{\mathrm{a}} / h$, the change of stiffness $k_{x, l}$ depending on $\eta$ is presented. The Fig. 7 shows the results of calculations for non-negative values $e_{\mathrm{a}} / h$ of 0 ; $0.05 ; 0.1 ; 0.167,0.2 ; 0.25$ and 0.3 . The constant length of the support of $l=1.0 \mathrm{~m}$ and the rectangular cross-section of the rod with the dimensions $b$ and $h$ is used in the calculations. It should be noted that for the small values of $e_{\mathrm{a}} / h$ from 0 to 0.1 and for small values $\eta$ (from 0.025 to 0.075 ), support stiffness is constant and corresponds to the support stiffness with flexural rigidity EI of the crosssection. Then, as the horizontal load is rising, the support stiffness begins to fall and decreases to zero. The value zero, for $e_{\mathrm{a}}=0$, reaches for $\eta=0.2$. The change of the parameter $\eta$ changes the schemes executed by the support. For the examples from the figure (Fig. 7), these are the following schemes: for $e_{\mathrm{a}} / h$ from 0 to $0.1-$ the schemes D2, E2; the scheme $E 2$ for $e_{\mathrm{a}} / h=0.167$ and for $e_{\mathrm{a}} / h$ smaller than 0.167 - the scheme $F$.

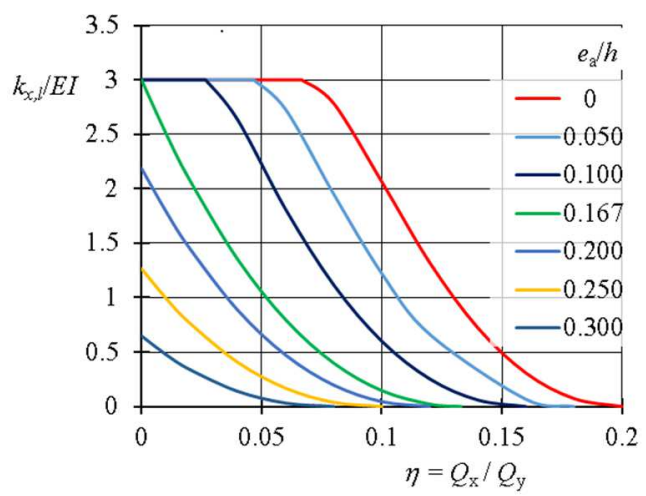

Fig. 7. The stiffness of the support $k_{x, l}$, as a value dependent on the horizontal load in case of $e_{\mathrm{a}} \geq 0$ : a) $0 \leq e_{\mathrm{a}} \leq h / 6$, b) $e_{\mathrm{a}} \geq$ $h / 6$.

The figure (Fig. 8a) shows the results of calculations for values $e_{\mathrm{a}} / h$ of $0,-0.05 ;-0.1 ;-0.167$; and figure (Fig. 8b) - for non-positive values $e_{\mathrm{a}} / h$ of $-0.167,-0.2 ;-0.25$; - 0.3 and 0 . Similar as previously, the constant support length was adopted of $l=1.0 \mathrm{~m}$ and the rectangular cross-section of the support. Similar as in the case $e_{\mathrm{a}} / h>0$, the support stiffness for small $e_{\mathrm{a}} / h$ of -0.167 to 0 is constant and corresponds to the support with the cross-section stiffness to bending of EI. Then, as the horizontal load is rising, the support stiffness begins to fall and decreases to zero. The value zero, for $e_{\mathrm{a}}=-0.167$, reaches for $\eta=0.27$. On the other hand, for high absolute values $e_{\mathrm{a}} / h$, of 0.2 and more, for the rising $\eta$, first growth in the stiffness values was 
noted, and then its decline. The change of the parameter $\eta$ changes the schemes executed by the support. For the examples from the figures (Fig. 8a), for $e_{\mathrm{a}} / h$ of -0.167 to - 0.05 , these are the following schemes: $D 1, E 1$; on the other hand, for $e_{\mathrm{a}} / h \leq-0.2$ (Fig. $8 \mathrm{~b}$ ), the schemes are: $A$, $B, C$.

a)

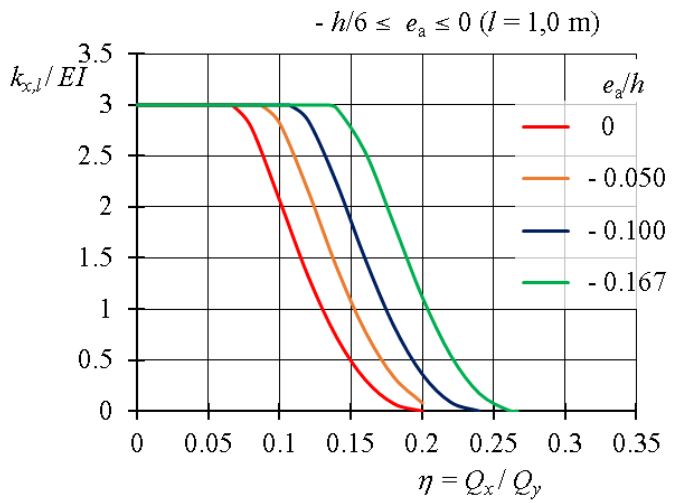

b)

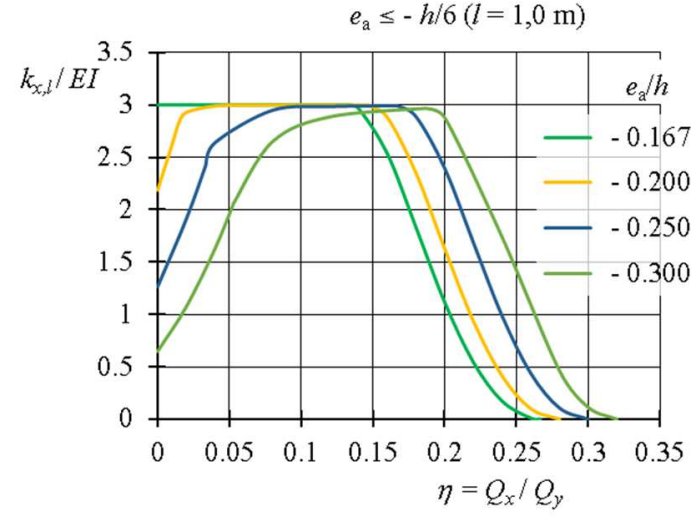

Fig. 8. The stiffness of the support $k_{x, l}$, as a value dependent on the horizontal load, for $e_{\mathrm{a}} \leq 0$ : a) $h / 6 \leq e_{\mathrm{a}} \leq 0$, b) $e_{\mathrm{a}} \leq h / 6$.

\section{Summary}

The temporary supports of buildings, consisting of a stack of unbounded repeatable elements, are loaded with the weight of the building applied eccentrically and with a horizontal force. Such load may lead to the existence of vertical positive deformations in the supports. Meanwhile, the supports, due to their construction, are not able to balance the normal stresses with values higher than zero. The stiffness of such supports, considering the lack of horizontal elements - for example bracings, is decisive for the safety of the buildings whose deflection is being eliminated.

An analytical rod model of a temporary support, consisting of a stack of unbounded elements, was defined. It was assumed in the model that the support material is not able to transmit positive stresses, and for negative stresses, it is characterised by linear properties. It was also assumed that the support is stocky and the load value is much smaller than a critical value. The effect of eccentricity at the rod displacement level has been omitted. The support model, depending on the value of the application eccentricity of the vertical load and on the value of horizontal load, may execute eight different static schemes. Dependencies describing model stiffness in the horizontal direction were introduced for all static schemes.

It was revealed that such stiffness depends on the quotient of the value between a horizontal and vertical load, and on the value and direction of eccentricity. If the horizontal load does not exist, and with the unintended eccentricity being within the cross-section core, the model stiffness corresponds to the cantilever rod. If eccentricity is increasing its absolute value, the support stiffness is falling.

If eccentricity does not exist, and if a horizontal load exists, the cross-section stiffness depends on the load value. For considerable values of the horizontal force, the support is executing a scheme of a rocker arm and its stiffness falls to zero. If a horizontal load and eccentricity exist at the same time, the support scheme and its stiffness depend on mutual directions and values of the horizontal load and eccentricity.

\section{References}

1. K. Gromysz, Methods of Removing Buildings Deflection Used In Poland. IOP Conf. Ser.: Mater. Sci. Eng. 245032096 (2017).

2. M.Y. Kabir, E. Shafei, Plasticity modelling of FRPconfined circular reinforced concrete columns subjected to eccentric axial loading. Composites: Part B 43 (2012) 3497-3506.

3. S.-D. Nie, S.-B. Kang, L. Shen, B. Yang, Experimental and numerical study on global buckling of Q460GJ steel box columns under eccentric compression. Engineering Structures 142 (2017) 211-222.

4. E. Bernat-Maso, L. Gil, P. Roca, Numerical analysis of the load-bearing capacity of brick masonry walls strengthened with textile reinforced mortar and subjected to eccentric compressive loading. Engineering Structures 91 (2015) 96-111.

5. E. Moradabadi, D.F. Laefer, J.A. Clarke, P.B. Lourenço, A semi-random field finite element method to predict the maximum eccentric compressive load for masonry prisms. Construction and Building Materials 77 (2015) 489-500.

6. M. Elchalakania, A. Karrecha, M. Dongao, M.S.M. Alib, B. Yangc, Experiments and Finite Element Analysis of GFRP Reinforced Geopolymer Concrete Rectangular Columns Subjected to Concentric and Eccentric Axial Loading. Structures 14 (2018) 273289.

7. E. Bernat-Maso, L. Gil, C. Escrig, Analysis of brick masonry walls strengthened with fibre reinforced polymers and subjected to eccentric compressive loads. Construction and Building Materials 84 (2015) 169-183.

8. L. Cavaleri, A. Failla, L.L. Mendola, M. Papia, Experimental and analytical response of masonry elements under eccentric vertical loads. Engineering Structures 27 (2005) 1175-1184. 